\title{
The biotechnological potential of Nannochloropsis
}

\author{
Umaima Al-Hoqani, Rosanna Young and Saul Purton*
}

Institute of Structural and Molecular Biology, University College London Gower Street, London, WC1E 6BT

United Kingdom

*Corresponding author: s.purton@ucl.ac.uk

With 2 figures and 5 tables

\begin{abstract}
Oleaginous microalgae have commercial potential as photoautotrophic cell factories capable of producing advanced biofuels and high-value speciality oils. One genus of particular interest is Nannochloropsis, which includes a number of robust marine species well suited to industrial-scale cultivation. Advances in bioprocess technology, together with strain enhancement through traditional mutagenesis or genetic engineering approaches, now offer the possibility of improving the economics of oil production from Nannochloropsis. In this review we describe the current and potential industrial applications of this genus, consider the present status of genetic enhancement methods, and highlight the need for new advances in this area - including the development of techniques for engineering the chloroplast genome.
\end{abstract}

Keywords: biofuels; eicosapentaenoic acid; genetic engineering; plastome; transformation; Nannochloropsis; Microchloropsis

\section{Introduction}

Nannochloropsis species are unicellular microalgae that belong to the class of Eustigmatophyceae within the Heterokontophyta and are recognised for their high photoautotrophic biomass productivity, their natural ability to accumulate high lipid content, and their successful cultivation at industrial scale (Radakovits et al. 2012). They are simple, non-flagellate, and spherical to slightly ovoid cells measuring 2-4 $\mu \mathrm{m}$ in size (Fig. 1A), making them difficult to distinguish from chlorophyte species (Sukenik 1999). Each cell has one or more yellow-green chloroplasts that occupy a significant part of the total cell volume and contain chlorophyll $a$ as the only chlorophyll. Violaxanthin is the main accessory pigment, with $\beta$-carotene, vaucheriaxanthin esters and several minor xanthophylls as additional accessory pigments (van den Hoek et al. 1995). The chloroplast is complex compared to that of chlorophyte algae because it is surrounded by four membranes derived from the secondary endosymbiosis of a red alga (Janouskovec et al. 2010). The outermost plastid membrane is connected with the outer nuclear envelope membrane to form a nucleus-plastid continuum (Murakami \& Hashimoto 2009) as illustrated in Figure 1B. Data from the genome sequencing projects and from NMR studies suggest that Nannochloropsis cell walls are cellulosic and contain sulphated fucans (Arnold et al. 2014, Corteggiani Carpinelli et al. 2014).
The Nannochloropsis genus is traditionally recognised as comprising the six species Nannochloropsis gaditana, Nannochloropsis salina, Nannochloropsis oculata, Nannochloropsis granulata, Nannochloropsis oceanica and Nannochloropsis limnetica (Fig. 1C) (Murakami \& Hashimoto 2009). However, a recent study based mainly on rbcL and 18S rDNA sequencing data (Fawley et al. 2015) has proposed that a new species, Nannochloropsis australis, be added and that $N$. gaditana and $N$. salina should be reclassified into a new genus named Microchloropsis. Another study suggested that $N$. gaditana could be reclassified as a strain of $N$. salina, owing to the $98.4 \%$ nucleotide identity and identical gene synteny between the two chloroplast genomes (Starkenburg et al. 2014). Reliable organellar phylogenetic markers for the inter- or intra-species phylotyping of Nannochloropsis have recently been determined by Wei et al. (2013) using systematic analysis of full organellar genome sequences. All Nannochloropsis species are found in marine environments except Nannochloropsis limnetica, which is found in fresh and brackish water (Jinkerson et al. 2013).

The high lipid productivity, abundance of polyunsaturated fatty acids and robust growth of Nannochloropsis species, together with the availability of genome sequences and molecular-genetic tools for various strains, make this genus attractive as cell platforms for the production of lipid molecules of industrial interest. Here we review the potential of Nannochloropsis in the aqua feed, food and green energy 

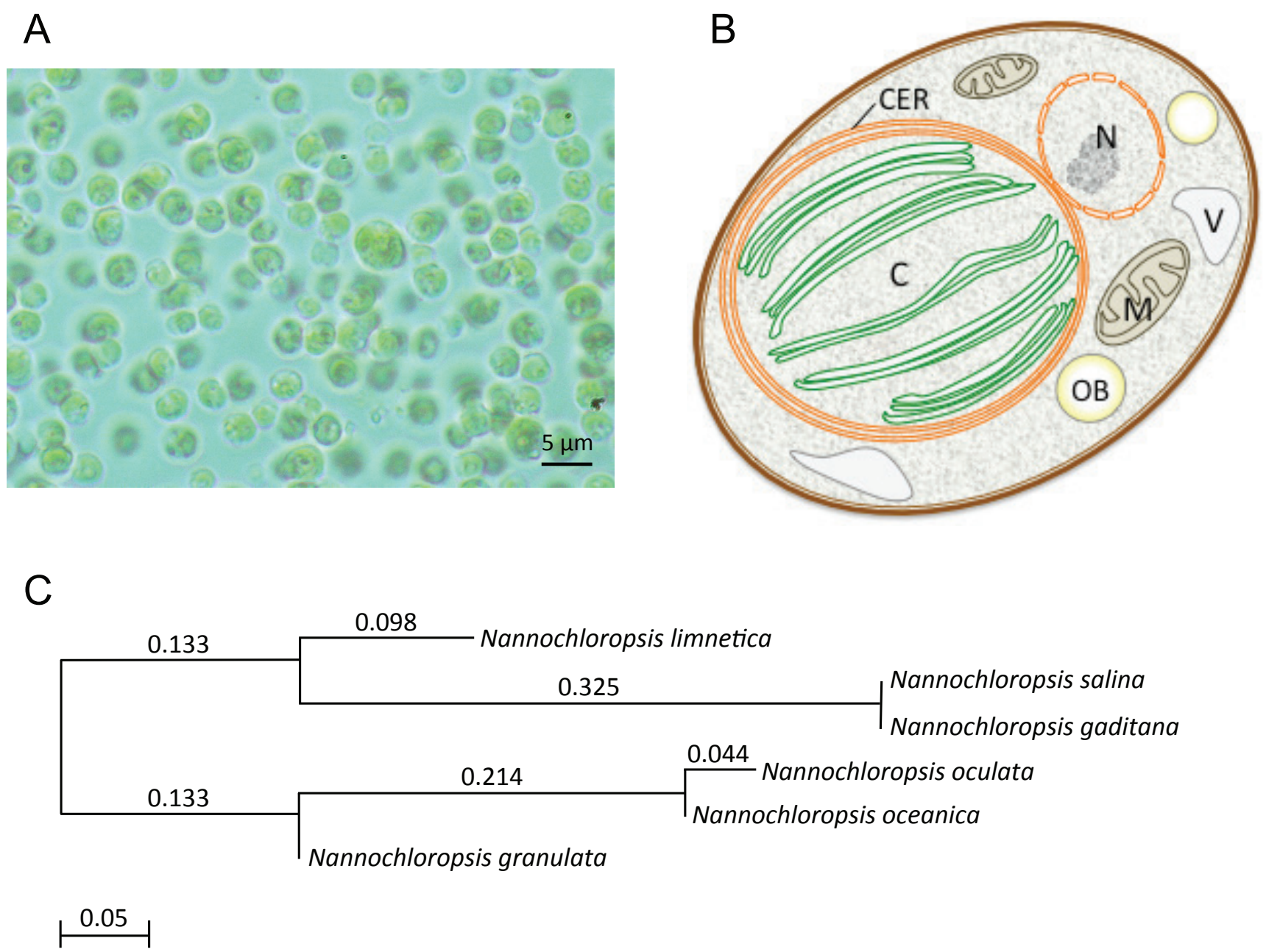

Fig. 1. (A) Light micrograph of Nannochloropsis oceanica under 100X magnification. (B) Illustrative cell structure for the Nannochloropsis genus under nutrient replete conditions, adapted from a figure by Lubián (1982). Abbreviations are C chloroplast, CER chloroplast endoplasmic reticulum; $\mathbf{M}$ mitochondrion; $\mathbf{N}$ nucleus; $\mathbf{O B}$ oil body; $\mathbf{V}$ vesicle. Under nutrient limitation stress, the oil body volume within the cell increases significantly (see, for example, Simionato et al. 2013). (C) Rooted neighbour-joining phylogenetic tree showing the relationship between different Nannochloropsis species based on whole alignment of their chloroplast genomes. Other phylogenetic trees for Nannochloropsis are based on 18S rDNA (Radakovits et al. 2012, Vieler et al. 2012); on 18S rDNA and rbcL (Fawley et al. 2015) and the mitochondrial genome (Wei et al. 2013).

industries. We consider the present status of genetic enhancement methods, and highlight the need for new advances in genetic engineering, including the need for a reliable method for engineering the chloroplast genome.

\section{Biotechnological applications for Nannochloropsis}

Potential biotechnological applications for Nannochloropsis species are summarised in Figure 2A. At present, the predominant commercial use is as the base of the food chain in the aquaculture industry. Companies such as Algaspring,
Archimede, Greensea, Monzon Biotech, Phycopure and Proviron grow and sell wild-type Nannochloropsis as aquafeed for the cultivation of marine fish, molluscs and shrimps, or for the production of zooplankton such as rotifers that are in turn used to supply fish hatcheries and nurseries, with the feedstock supplied as concentrates of live algae, or as frozen or lyophilised algae (Lubzens et al. 1995, Camacho-Rodríguez et al. 2016). The main attraction of Nannochloropsis for the aquafeed industry is its favourable fatty acid composition, which includes a relatively high content of eicosapentaenoic acid (EPA) (Sukenik 1999, Ma et al. 2016). EPA is a highly unsaturated omega-3 fatty acid (20:5) and is a useful dietary component in preventing several human diseases. Although 
EPA is commonly called a 'fish oil', marine fish cannot synthesize this molecule and rely on a dietary source. For $N$. gaditana, EPA productivity can reach $30 \mathrm{mg} \mathrm{l}^{-1}$ day $^{-1}$ in outdoor photobioreactors (Camacho-Rodriguez et al. 2014) and can represent as much as $27 \%$ of total fatty acids under nutrient-sufficient conditions (Ferreira et al. 2009). The nutritional value of Nannochloropsis under different growth conditions and the transfer of their nutrients through food chains has been extensively investigated (Fernandez-Reiriz \& Labarta 1996, Ferreira et al. 2009, Camacho-Rodríguez et al. 2014), and the use of marine microalgae in the aquaculture industry has been the subject of several detailed reviews (Becker 2013, Gressel 2013).

The use of Nannochloropsis as an aquaculture feed also offers potential opportunities for creating transgenic lines for oral delivery of pharmaceutical proteins that improve fish growth rates and reduce loss through pathogens. Chen et al. (2008) reported significant improvements in growth of tilapia larvae when the feedstock of $N$. oculata was replaced with a transgenic line engineered to produce fish growth hormone. In a separate study, the same species was engineered to produce the anti-microbial peptide bovine lactoferricin. Feeding medaka fish with this transgenic line greatly improved their survival rates when subsequently infected with a bacterial pathogen (Li \& Tsai 2009). There is also interest in using microalgae such as Nannochloropsis for the oral delivery of antigens to the many viral, bacterial and fungal pathogens that plague the aquaculture industry, thereby providing a simple low-cost method of vaccine delivery (Siripornadulsil et al. 2007).

Exploitation of Nannochloropsis PUFA-rich feed is not limited to aquaculture and there is growing interest in its use in other animal feeds to improve the nutritional value of farmed foods. For example, a recent study demonstrated such improvement in the nutritional value of egg yolk by adding Nannochloropsis biomass to laying hens' feed (Lemahieu et al. 2013). Another growing sector is the production of microalgae for use directly in the human diet: Qualitas Health produces liquid capsules containing EPA-rich oil from wild type $N$. oculata grown in shallow ponds in Texas, while Optimally Organic sells $N$. gaditana dried powder as a nutritional supplement. The safety of both the oil from $N$. oculata and whole cells of the alga has been assessed in toxicology studies and determined safe for use as a dietary supplement (Kagan et al. 2014, Kagan \& Matulka 2015). Such products therefore represent vegetarian sources of EPA that avoid the problems of declining fish stocks and potential heavy metal contaminants found in fish oils.

The development of Nannochloropsis as a feedstock for biofuel production has also been the focus of much research over the past decade, but there are some key issues to be addressed before algal biofuel production becomes cost effective and competitive with current fuel supplies (Umdu et al. 2009, Doan \& Obbard 2015, Zhu et al. 2014, Ma et al. 2014, Hu et al. 2015). These include: i) developing strains that produce high quantities of triacylglycerols (TAGs) with the desired chain lengths and degree of saturation for conversion to fungible biofuels (Taleb et al. 2015); ii) understanding the link between growth conditions and lipid productivity; iii) developing large-scale cultivation facilities (Fig. 2B), and iv) refining oil extraction techniques. In addition to TAGs, alka(e)nes including heptadecane, heptadecene and pentadecane were recently identified in several Nannochloropsis species (Sorigué et al. 2016) and may be suitable for inclusion in jet fuels and diesel fuels.

Fossil fuels and first-generation biofuels derived from land-based energy crops are already available on the market in large quantities, whereas commercial production of algaederived biofuels are expected to require more advanced technologies (Hannon et al. 2010, Medipally et al. 2015). One aspect of this is the economics of large-scale, outdoor cultivation - as illustrated by Vree et al. (2015) in a comparative study of Nannochloropsis growth in four different production systems. The highest areal productivities were achieved in a closed vertical photobioreactor and the lowest in an open pond system, whilst the capital costs for the former are considerably higher than the latter.

\section{Enhancing the lipid profile in Nannochloropsis: strain choice, growth conditions and mutant selection}

Several species in the genus Nannochloropsis are recognized as oleaginous algae owing to their ability to accumulate large quantities of lipid. However, there is inter- and intra-species variation in the lipid productivity and fatty acid composition (Ma et al. 2014, Beacham et al. 2014). In a study of nine Nannochloropsis strains, specific growth rates were found to range from 0.07 to 0.21 day $^{-1}$ while the lipid content varied from 37 to $60 \%$ of dry weight (Ma et al. 2014). The predominant fatty acids in most of these strains were 16:0, 16:1 and 18:1. The most suitable strain depends on the desired product; strains for fish oil production should have high levels of EPA either in polar lipids for good bioavailability or as neutral TAGs for ease of extraction, whereas those for biodiesel need shorter saturated and monounsaturated fatty acids in TAGs. Significant variation is also seen in cell wall thickness among Nannochloropsis species, which could impact on both the efficiency and cost of lipid extraction, as well as the ease with which a strain can be genetically engineered (Beacham et al. 2014). Furthermore the salinity of the culture medium also influences the thickness of the wall within a species, so growth conditions could be optimized to favour downstream processing (Beacham et al. 2014).

Growth conditions are also key to maximizing lipid yield. Nitrogen deprivation and other stress conditions are typically used to induce increased lipid content in Nannochloropsis and other microalgae. However, these conditions result in impeded cell growth and photosynthesis, affecting the 

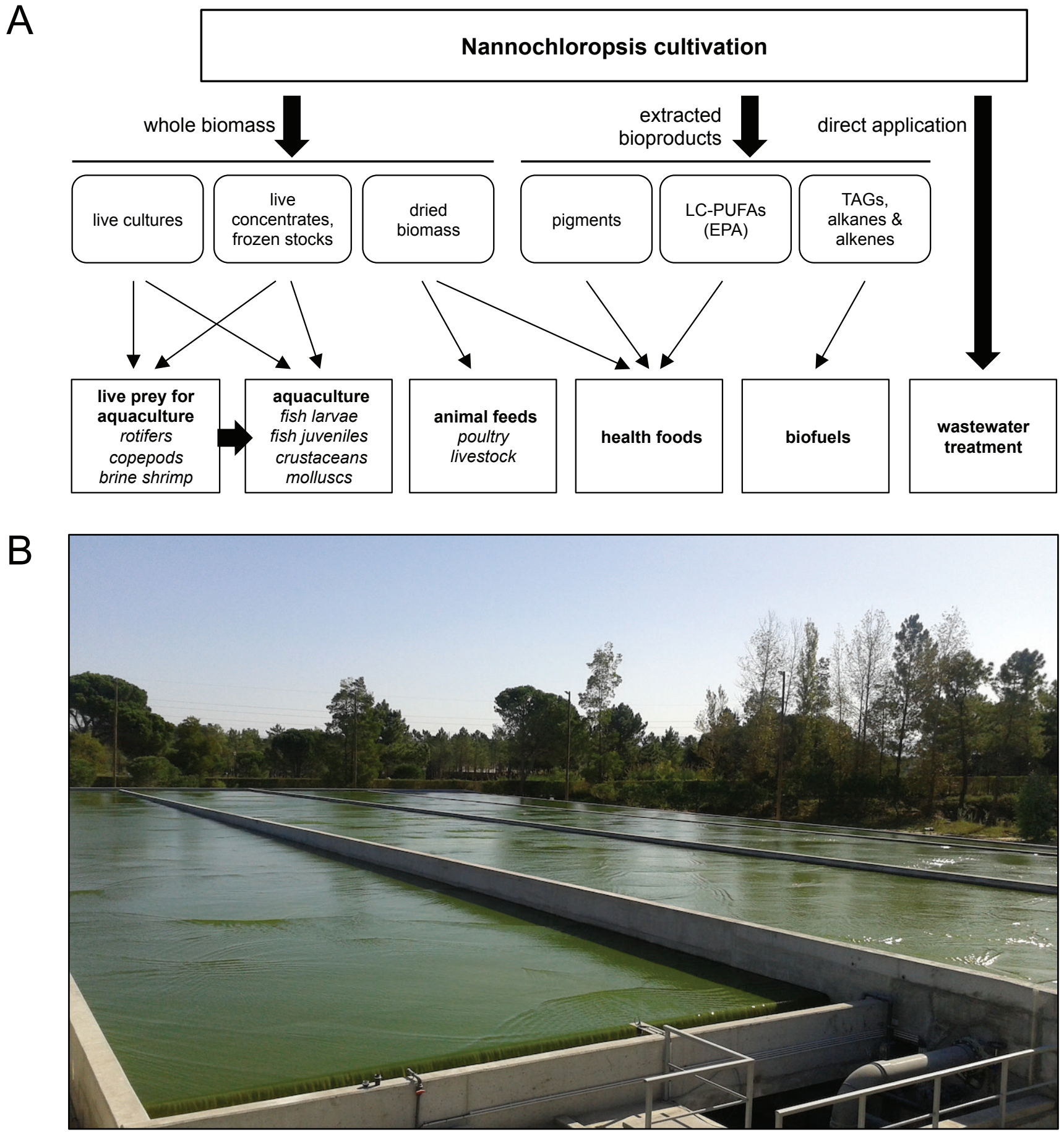

Fig. 2. (A) Current and potential biotechnological applications of Nannochloropsis. (B) Cascade raceway used in the E.U. BIOFAT project for Nannochloropsis cultivation (reproduced with permission from BIOFAT, www.biofat-project.eu).

biomass productivity and making the system less commercially viable (Radakovits et al. 2012, Corteggiani Carpinelli et al. 2014). The nitrogen starvation response has been studied in detail and includes a reorganization of the photosynthetic apparatus (Simionato et al. 2013). There have been several attempts to reach high lipid content without losing high biomass productivity, using for instance a twostage cultivation process ( $\mathrm{Su}$ et al. 2011) and conventional mutagenesis approaches (Schneider et al. 1995, Beacham et al. 2015). Franz et al. (2013) screened small molecules for their ability to increase intracellular lipid levels as measured by Nile red staining, finding that quinacrine was effective 
for N. oculata and epigallocatchetin gallate for N. salina. In addition, exposure to UV-C radiation was found to lead to a two-fold increase in total EPA content in Nannochloropsis sp. cultures (Sharma \& Schenk 2015).

Following the selection of robust lipid producer strains among Nannochloropsis species and adjusting growth conditions to achieve the best yield, genetic approaches can be used to further optimize a strain: i.e. domesticating a wild isolate through phenotypic improvements that make it more suited to industrial application. The simplest strategy is a 'forward genetics' approach involving random mutagenesis by classical physical or chemical methods, followed by the selection of strains with higher lipid productivity or other desirable phenotypes. This genetic approach is aided by the fact that Nannochloropsis species appear to be haploid (Kilian et al. 2011), and therefore both dominant and recessive mutations display a phenotype. However, there are as yet no reports of sexual reproduction in Nannochloropsis, and therefore classical breeding programmes aimed at combining desirable traits, eliminating undesired mutations from strains, and mapping mutations may not possible. Genome sequencing failed to identify meiosis-specific genes in the $N$. oceanica genome (Pan et al. 2011) although, actively transcribed meiosis-specific genes were reported for $N$. gaditana B-31 (Corteggiani Carpinelli et al. 2014). The capacity for sexual reproduction, if present at all, may therefore vary between Nannochloropsis species and may require more than one mating type. Nonetheless, mutagenesis screens have led to the successful isolation of mutant strains with smaller antenna size, and therefore increased light-use efficiency under bulk cultivation conditions (Perin et al. 2015). In addition, there are a number of reports of strain improvements in lipid profile or productivity through classical mutagenesis as summarized in Table 1.

\section{Progress on genetic engineering of the Nannochloropsis nuclear genome}

Whilst forward genetic screens can lead to enhanced phenotypes, the improvement of Nannochloropsis strains for industrial applications also requires robust and routine technologies for genetic engineering. These then allow 'reverse-genetic' strategies in which endogenous genes are manipulated, or foreign genes introduced into the genome to give desired new phenotypes. Most efforts to date have focused on genetic engineering of the nuclear genome, as discussed in this section. However, the development of complementary techniques for engineering the chloroplast genome is also necessary, as discussed in the subsequent section. The first successful nuclear transformation of Nannochloropsis was reported by Chen et al. (2008), who used electroporation of protoplasts to introduce a gene encoding fish growth hormone under the control of an inducible promoter. Subsequently, reports appeared describing the nuclear transformation of Nannochloropsis without cell wall removal using either Agrobacterium (Cha et al. 2011), electroporation (Kilian et al. 2011) or microparticle bombardment (Kang et al. 2015a, c). A number of selectable markers and reporter genes have been developed that allow selection of transformant lines and assays of transgene expression levels (Table 2). The most effective markers to date are those that confer resistance against antibiotics such as zeocin (the Sh.ble gene) and hygromycin B (aph7). On the other hand, two native genes that are required for growth on nitrate as the sole nitrogen source have been successfully knocked out in one Nannochloropsis strain (Kilian et al. 2011), opening up the possibility of using the genes as endogenous selectable markers. The nitrate reductase and nitrite reductase knockout cell lines cannot grow on nitrate as the nitrogen source but can be maintained on medium containing ammonium. Hence, re-introduction of the gene into the corresponding mutant should allow selection on nitrate.

Another marker that has been expressed successfully in Nannochloropsis is the purple chromoprotein gene from Stichodacyla haddoni (shCP), which is not directly selectable but produces a distinctive brown phenotype that can be easily identified in the background of non-transformed cells (Shih et al. 2015). Reporter genes such as the $\beta$-glucuronidase gene (GUS) and adapted versions of the gene for green fluorescent protein (GFP) have been used to test promoters and transformation techniques in Nannochloropsis (Cha et al. 2011, Moog et al. 2015). In addition, the first in vivo localization study of Nannochloropsis has been reported by Moog et al. (2015) using GFP, highlighting the possibility of using $\mathrm{N}$-terminal targeting sequences to target nuclear-encoded proteins of interest to different cellular compartments such as the nucleus, mitochondria, endoplasmic reticulum or chloroplast. A recent study has developed a reporter gene to overcome the interference of autofluorescent signals from cells and maintain greater brightness with photostability by using the genetically modified mCherry fluorescent protein "sfCherry fluorescent" (Kang et al. 2015a).

Interestingly, a study shows that homologous recombination in the Nannochloropsis sp. W2J3B nucleus can occur when transgenes are flanked with homologous genomic sequence (Kilian et al. 2011). This could allow both the precise and predictable insertion of transgenes into defined nuclear loci, and the systematic manipulation and functional analysis of specific endogenous genes. However, efficient integration of exogenous DNA into the nuclear genome via homologous recombination has yet to be reported in any other Nannochloropsis strain, and it appears that for most transformation events in other strains the transgenes insert into the genome at apparently random loci, and sometimes in multiple copies. This can lead to 'position effects' in which the level and stability of transgene expression varies between transformant lines. For overexpression studies or the introduction of foreign genes, the introduction of episomal plasmids via bacterial conjugation may be an option; such a 


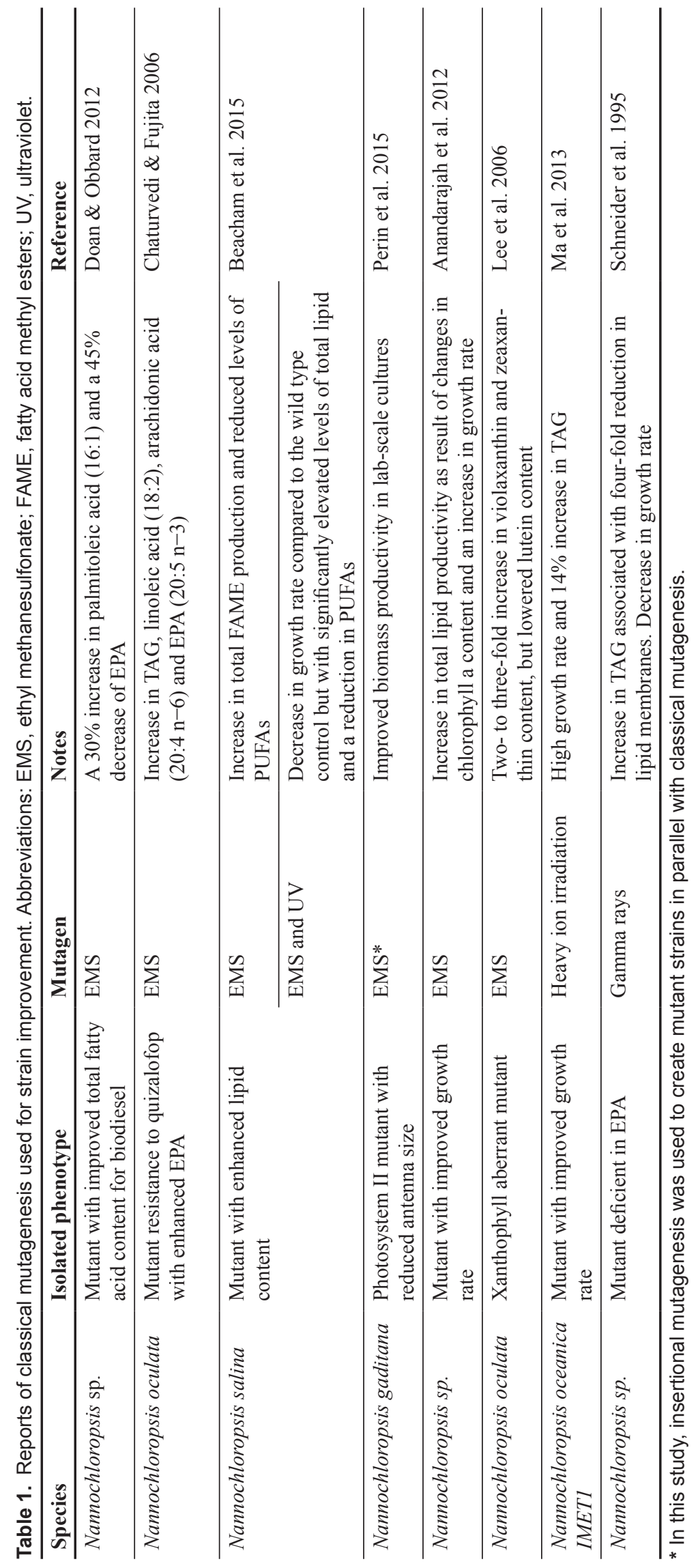




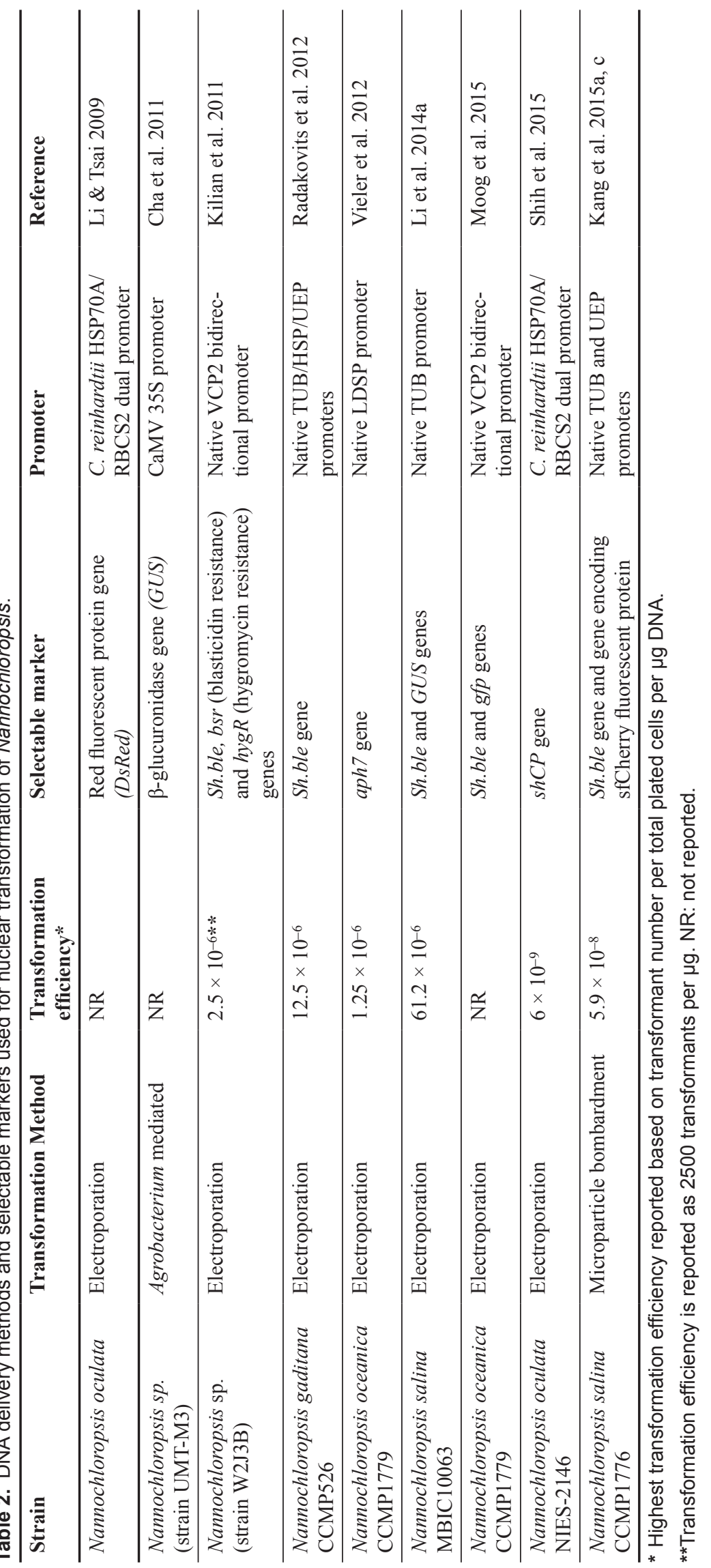




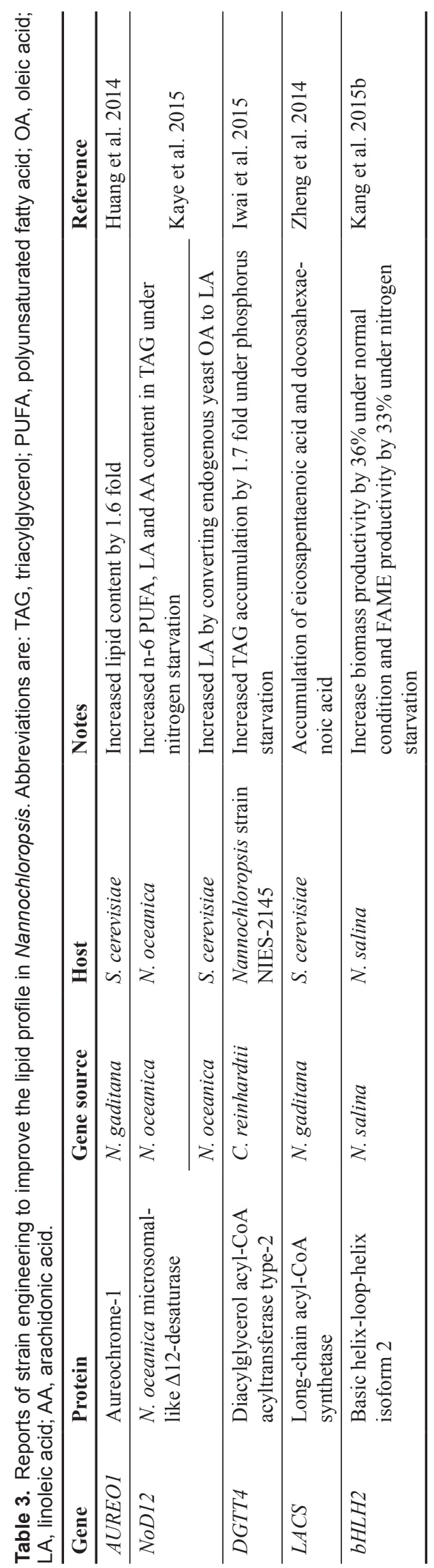


Table 4. Reports of chloroplast transformation in microalgae.

\begin{tabular}{lllll}
\hline Algal species & Transformation method & Selectable marker & Selection & References \\
\hline Chlamydomonas reinhardtii & Particle gun & atpB & Photoautotrophy & Boynton et al. 1988 \\
\cline { 2 - 5 } & Glass beads & tscA, atpB & Photoautotrophy & Kindle et al. 1991 \\
\hline Haematococcus pluvialis & Particle gun & aadA & Spectinomycin & Gutiérrez et al. 2012 \\
\hline Dunaliella tertiolecta & Particle gun & ereB & Erythromycin & Georgianna et al. 2013 \\
\hline Platymonas subcordiformis & Particle gun & bar & Basta & Cui et al. 2014 \\
\hline Porphyridium sp. & Particle gun & AHAS (W492S) & Sulfometuron methyl & Lapidot et al. 2002 \\
\hline Euglena gracilis & Particle gun & aadA & Streptomycin and & Doetsch et al. 2001 \\
& & & spectinomycin & \\
\hline Phaeodactylum tricornutum & Electroporation & cat & Chloramphenicol & Xie et al. 2014 \\
\hline
\end{tabular}

system has recently been developed for diatoms (Karas et al. 2015).

Strain engineering for increased lipid productivity requires knowledge of the relevant biosynthetic pathways so that particular genes can be chosen for knockout, overexpression or introduction. The identification and characterization of lipid metabolic pathway genes, including those involved in fatty acid biosynthesis, TAG assembly, lipid activation and degradation could be used as a guide for rational genetic engineering of Nannochloropsis. Several research groups have sequenced and annotated the genomes of different Nannochloropsis species and in some cases investigated their metabolic pathways (Radakovits et al. 2012, Vieler et al. 2012, Corteggiani Carpinelli et al. 2014, Wang et al. 2014), providing a rapid and effective way to gain the basic knowledge for further studies. The integration of available genomic data with transcriptome (Tian et al. 2013, Zheng et al. 2013), proteome (Simionato et al. 2013) and lipidome ( $\mathrm{Li}$ et al. 2014b) data of various Nannochloropsis species assists in elucidating the genes involved and how transcriptional changes modulate increased metabolic flux within the biosynthesis pathways under a variety of physiological growth conditions. Further utilization of these data for modelling and overexpression studies will provide a better understanding of lipid biosynthesis in oleaginous algae and opportunities for increasing the production of specific lipids through metabolic engineering. Although the overexpression of genes involved in lipid biosynthesis does not always lead to the predicted outcomes (La Russa et al. 2012), recent transgenic studies have resulted in increased TAG content in Nannochloropsis, or the elucidation of gene function through the over-expression of Nannochloropsis genes in the model yeast, Saccharomyces cerevisiae (Table 3). Other genetic engineering approaches that should be considered for increased lipid production include increasing photosynthetic efficiency under nitrogen deprivation, blocking competing pathways and reduction of TAG catabolism, and decoupling TAG accumulation and stress conditions such as nitrogen deprivation (Klok et al. 2014).

\section{Perspectives for chloroplast genomic engineering}

The chloroplast is the site of primary energy production within the algal cell and also houses key metabolic pathways such as those involved in the biosynthesis of carbohydrates, fatty acids, tetrapyrroles and terpenes. The full exploitation of Nannochloropsis and other microalgae as a biotechnology platform for biofuel production or synthesis of high-value metabolites therefore requires the development of methods for engineering the chloroplast genome (=plastome). This would allow the manipulation of endogenous chloroplast genes involved in energy transduction and carbon fixation, and the introduction of foreign genes encoding novel metabolic enzymes (Purton et al. 2013). Progress is being made in the development of chloroplast transformation methodology for a number of microalgal species (Table 4). However there are no reports as yet of successful chloroplast transformation of Nannochloropsis or other microalgae that harbour secondary plastids, with the exception of Phaeodactylum tricornutum (Xie et al. 2014). In this section we consider the three key prerequisites for achieving chloroplast transformation in Nannochloropsis.

(i) Prerequisite 1: plastome sequence for the chosen species Integration of DNA into the plastome occurs via homologous recombination, allowing site-directed modification and the precise insertion of foreign DNA into predetermined loci. Consequently, prior knowledge of the plastome sequence is required in order to manipulate the target chloroplast gene or flank foreign DNA with homologous elements. Furthermore, successful expression of foreign genes typically requires the use of endogenous genetic elements such as promoters and untranslated regions (Purton et al. 2013). Wei et al. (2013) sequenced plastomes from at least one strain of each Nannochloropsis species; the plastomes were found to range from $115-118 \mathrm{~kb}$ in size, contain $123-126$ predicted protein-coding genes and had a GC content in the range of $33.0-33.6 \%$. The chloroplast genome sequences of other 
strains are also publicly available (Radakovits et al. 2012, Corteggiani Carpinelli et al. 2014, Starkenburg et al. 2014). These data provide the starting point for the design of chloroplast genetic engineering strategies.

(ii) Prerequisite 2: a suitable selection system

Development of a successful chloroplast transformation protocol relies on the availability of an effective selectable marker gene that facilitates the growth of transformant cells on selective media. Traits that have been widely used in algal chloroplast transformation as selection methods include restoration of photoautotrophy, resistance to antibiotics, tolerance to herbicides, and the complementation of metabolic mutants (reviewed by Potvin \& Zhang 2010, Day \& Goldschmidt-Clermont 2011). Heterotrophic growth using glucose or ethanol as an organic carbon source has been reported for one Nannochloropsis strain (Fang et al. 2004), suggesting that chloroplast mutants defective in photosynthesis could be isolated and the corresponding wild-type gene used as a marker to restore photoautotrophy. However, no non-photosynthetic mutants have yet been described nor have any auxotrophic mutants been described that are defective in a key metabolic pathway within the organelle. Antibiotics and herbicides that target aspects of chloroplast biology have been screened by ourselves (unpublished data: see Table 5) and others (Vieler et al. 2012, Chernyavskaya 2014) for their effect on various Nannochloropsis species. Most compounds tested were found to have little effect on cell growth even at high concentrations. This could be due to the complexity of plastid membranes, preventing easy access for those molecules into the chloroplast stroma and thus failing to exhibit an inhibitory effect. However, chloramphenicol and the photosystem II inhibitors, DCMU and atrazine show promise as selective agents (Table 5), as does paromomycin for $N$. oceanica but not for other Nannochloropsis species (Vieler et al. 2012).

(iii) Prerequisite 3: A method to introduce exogenous DNA into the chloroplast

DNA delivery into the algal chloroplast has been achieved using microparticle bombardment (= biolistics), glass bead agitation and electroporation (Table 4). Biolistics is generally a reliable method for delivering DNA across cell walls and multiple membranes, and has been employed successfully for nuclear transformation in many algal species (Gangl et al. 2015). However, progress in achieving chloroplast transformation is still limited to relatively few species, not least because of the small cell size of many algae. The gold or tungsten microparticles used for biolistic DNA delivery are typically $0.5-1.7 \mu \mathrm{m}$ in diameter, which is rather large in comparison to a Nannochloropsis cell (approximately 2-4 $\mu \mathrm{m}$ in diameter), with the chloroplast compartment being even smaller. Recently, gold 'nanoparticles' with a diameter of $40 \mathrm{~nm}$ have become available and may be more suitable; O'Brien \& Lummis (2011) demonstrated that these caused much less tissue damage than $1 \mu \mathrm{m}$ gold particles during the

Table 5. Results from our group on the effect of selected compounds on N. gaditana CCMP526. ">" indicates the highest concentration of the respective compound tested.

\begin{tabular}{|c|c|c|c|}
\hline Compound & Mode of action & Inhibitory level $(\mu \mathrm{g} / \mathrm{ml})$ & $\begin{array}{l}\text { Selectable marker or dominant } \\
\text { allele conferring resistance }\end{array}$ \\
\hline \multicolumn{4}{|l|}{ Antibiotics } \\
\hline Chloramphenicol & Inhibitor of $70 \mathrm{~S}$ ribosome & 50 & catA1 \\
\hline Kanamycin & Inhibitor of $70 \mathrm{~S}$ ribosome & $>200$ & aphA6 \\
\hline Spectinomycin & Inhibitor of $70 \mathrm{~S}$ ribosome & $>200$ & $\operatorname{aad} A$ \\
\hline Streptomycin & Inhibitor of 70S ribosome & $>200$ & $\operatorname{aad} A$ \\
\hline \multicolumn{4}{|l|}{ Herbicides } \\
\hline Harvest & $\begin{array}{l}\text { Inhibitor of glutamine synthetase } \\
\text { activity }\end{array}$ & $>100$ & pat \\
\hline Glufosinate-ammonium & $\begin{array}{l}\text { Inhibitor of glutamine synthetase } \\
\text { activity }\end{array}$ & $>500$ & pat \\
\hline Bialaphos & $\begin{array}{l}\text { Inhibitor of glutamine synthetase } \\
\text { activity }\end{array}$ & $>350$ & pat \\
\hline Diuron (DCMU) & Inhibitor of photosystem II & 30 & Point mutation in $p s b A$ \\
\hline Atrazine & Inhibitor of photosystem II & 50 & $a t z A$ or point mutation in $p s b A$ \\
\hline \multicolumn{4}{|l|}{ Amino acids } \\
\hline D-alanine & $\begin{array}{l}\text { Interferes with amino acid } \\
\text { metabolism }\end{array}$ & $>400$ & dao \\
\hline
\end{tabular}


biolistic transformation of human and mouse cells and were equally as efficient.

Electroporation has also been used successfully for nuclear transformation for a wide range of microalgae, both with and without cell walls (Radakovits et al. 2012, Zhang \& $\mathrm{Hu}$ 2013). This method relies on subjecting the cells to controlled electrical pulses of high charges for a short period of time (ms), which results in temporary pores in the cell membranes through which the exogenous DNA enters the cells. The reported transformation of the P. tricornutum plastid with the cat gene by electroporation (Xie et al. 2014) opens up the possibility of using this technique for other algal species whose chloroplasts are surrounded by multiple membranes, such as Nannochloropsis.

Given the current lack of methodology to transfer genes directly into the Nannochloropsis chloroplast genome, an alternative strategy is to transform the nucleus with genes encoding chloroplast-targeted proteins. This could give insights into industrially relevant biosynthetic pathways that take place in chloroplast and may also enable the introduction of novel pathways. There are relatively few studies in heterokont algae reporting success in targeting foreign proteins into the chloroplast using endogenous $\mathrm{N}$-terminal signal and transit peptides, and more research is required in this direction (Gruber et al. 2007, Sunaga et al. 2014, Moog et al. 2015).

\section{Conclusions and future directions}

Nannochloropsis species have great potential as environmentally sustainable sources of biofuels and nutritionally important oils such as long chain omega-3 fatty acids. However, in order to make these products economically viable and competitive, there is a need for both biological improvements to the strains used and the development of more cost-effective and energy efficient bioprocessing technologies including cultivation, harvesting and product preparation. This is particularly true for biofuels where the desired product is high volume/low value. Many recent studies have examined the possibility of using municipal wastewater or industrial effluent to grow Nannochloropsis species for biomass production as part of an integrated system of wastewater treatment. For example, $N$. oculata grows well in $20 \%$ untreated municipal wastewater diluted in seawater, resulting in the successful removal of $80 \%$ of the nitrogen and phosphorus (Sirin $\&$ Sillanpaa 2015). Since lipid productivity is influenced by two conflicting factors - nutrient availability (to maximize biomass production) and nutrient deprivation (to increase TAG accumulation), a balance must be struck between a sufficient rate of wastewater treatment, biomass accumulation and lipid accumulation (as demonstrated for $N$. salina by Cai et al. (2013)).

Strain engineering would also help to address such challenges by creating strains with enhanced TAG accu- mulation under nutrient replete conditions. To do this, we must build on our current understanding of lipid metabolic/catabolic pathways and how these are influenced by growth conditions such as nutrient concentration, salinity, temperature and light. Progress in this direction is starting to be made using systems biology approaches to develop dynamic models of lipid metabolism in Nannochloropsis and related algal groups (e.g. Dong et al. 2013, Mühlroth et al. 2013), and to understand at the genome level the transcriptional factors that regulate gene expression $(\mathrm{Hu}$ et al. 2014). In addition to such models, a set of molecular tools is needed to enable the genetic manipulation of lipid biology in both the nucleus and plastid, and to modify other aspects of Nannochloropsis physiology to enhance performance and productivity under industrial cultivation conditions. For example, Lu \& Xu (2015) have proposed that increased biomass productivity and elevated tolerance to abiotic stresses could be achieved by manipulation of endogenous phytohormone levels in the algae. Such multitrait strain improvement strategies require advanced techniques for predictable and precise genome engineering. New nuclear genome editing techniques that are being applied successfully in other organisms (Hsu et al. 2014, Chandrasegaran \& Carroll 2016) would certainly advance the field. These editing approaches rely on double-stranded breaks made at targeted loci using bespoke nucleases such as engineered meganucleases, zinc finger nucleases, transcription activator-like effector nucleases (TALENs) or the CRISPR/Cas9 system, and are still very much in the early stages of development for microalgae. However, three recent reports of successful targeted insertions and gene knockouts in the diatom Phaeodactylum tricornutum using meganucleases, TALENs and CRISPR-Cas9, respectively (Daboussi et al. 2014; Weyman et al. 2015; Nymark et al. 2016) should encourage efforts to develop these technologies for other heterokont algae such as Nannochloropsis. Similarly, further studies of how foreign DNA integrates into the Nannochloropsis nuclear genome will reveal whether targeted integration via homologous recombination is limited to one or a few species, or could be applied more generally as a genome engineering tool (Weeks 2011). The successful transformation of the P. tricornutum plastid (Xie et al. 2014) suggests also that the hurdles for engineering secondary plastids can be overcome, allowing a full suite of technologies for making designer strains of Nannochloropsis.

Acknowledgments: We thank University College London for covering the publication costs. Also, we are grateful to Diana Fonseca from the BIOFAT project for providing the photograph in figure 2. UA-H is funded by a doctoral scholarship from the Ministry of Manpower, Sultanate of Oman. Research on Nannochloropsis in the Purton group is funded by grant BB/L002957/1 from the UK's Biotechnology and Biological Sciences Research Council (BBSRC). 


\section{References}

Anandarajah, K., Mahendraperumal, G., Sommerfeld, M. \& Hu, Q. (2012): Characterization of microalga Nannochloropsis sp. mutants for improved production of biofuels. - Appl. Energy 96: 371-377.

Arnold, A.A., Genard, B., Zito, F., Tremblay, R., Warschawski, D.E. \& Marcotte, I. (2014): Identification of lipid and saccharide constituents of whole microalgal cells by $13 \mathrm{C}$ solid-state NMR. - Biochim. Biophys. Acta - Biomembr. 1848: 369-377.

Beacham, T.A., Bradley, C., White, D.A., Bond, P. \& Ali S.T. (2014): Lipid productivity and cell wall ultrastructure of six strains of Nannochloropsis: Implications for biofuel production and downstream processing. - Algal Res. 6: 64-69.

Beacham, T.A., Macia, V.M., Rooks, P., White, D.A. \& Ali, S.T. (2015): Altered lipid accumulation in Nannochloropsis salina CCAP849/3 following EMS and UV induced mutagenesis. Biotechnol. Reports 7: 87-94.

Becker, E.W. (2013): Microalgae for Aquaculture: Nutritional Aspects. - In: Richmond, A. \& Hu, Q. (ed.), Handbook of Microalgal Culture: Applied Phycology and Biotechnology, pp. 671-691. - John Wiley \& Sons, Ltd., Chichester.

Boynton, J.E., Gillham, N.W., Harris, E.H., Hosler, J.P., Johnson, A.M., Jones, A.R., Randolph-Anderson, B.L., Robertson, D., Klein, T.M., Shark, K.B. \& Sanford, J.C. (1988): Chloroplast transformation in Chlamydomonas with high velocity microprojectiles. - Science 240: 1534-1538.

Cai, T., Park, S.Y., Racharaks, R. \& Li, Y. (2013): Cultivation of Nannochloropsis salina using anaerobic digestion effluent as a nutrient source for biofuel production. - Appl. Energy 108: 486-492.

Camacho-Rodríguez, J., Cerón-García, M.C., Macías-Sánchez, M.D., Fernández-Sevilla, J.M., López-Rosales, L. \& MolinaGrima, E. (2016): Long-term preservation of concentrated Nannochloropsis gaditana cultures for use in aquaculture. J. Appl. Phycol. 28: 299-312.

Camacho-Rodríguez, J., González-Céspedes, A.M., Cerón-García, M.C., Fernández-Sevilla, J.M., Acién-Fernández, F.G. \& Molina-Grima, E. (2014): A quantitative study of eicosapentaenoic acid (EPA) production by Nannochloropsis gaditana for aquaculture as a function of dilution rate, temperature and average irradiance. - Appl. Microbiol. Biotechnol. 98: 2429-2440.

Cha, T.S., Chen, C.F., Yee, W., Aziz, A. \& Loh, S.H. (2011): Cinnamic acid, coumarin and vanillin: Alternative phenolic compounds for efficient Agrobacterium-mediated transformation of the unicellular green alga, Nannochloropsis sp. J. Microbiol. Methods 84: 430-434.

Chandrasegaran, S. \& Carroll, D. (2016). Origins of programmable nucleases for genome engineering. - J. Mol. Biol. 428: 963-989.

Chaturvedi, R. \& Fujita, Y. (2006): Isolation of enhanced eicosapentaenoic acid producing mutants of Nannochloropsis oculata ST-6 using ethyl methane sulfonate induced mutagenesis techniques and their characterization at mRNA transcript level. Phycol. Res. 54: 208-219.

Chen, H.L., Li, S.S., Huang, R. \& Tsai, H.J. (2008): Conditional production of a functional fish growth hormone in the transgenic line of Nannochloropsis oculata (Eustigmatophyceae). J. Phycol. 44: 768-776.

Chernyavskaya, O. (2014): Developing molecular tools for the genetic manipulation of Nannochloropsis. - MSc thesis. Norges
Teknisk-Naturvitenskapelige Universitet, Norway. [http://brage. bibsys.no/xmlui/handle/11250/246154]

Corteggiani Carpinelli, E., Telatin, A., Vitulo, N., Forcato, C., D’Angelo, M., Schiavon, R., Vezzi, A., Giacometti, G.M., Morosinotto, T. \& Valle, G. (2014): Chromosome scale genome assembly and transcriptome profiling of Nannochloropsis gaditana in nitrogen depletion. - Mol. Plant 7: 323-335.

Cui, Y., Qin, S. \& Jiang, P. (2014): Chloroplast transformation of Platymonas (Tetraselmis) subcordiformis with the bar gene as selectable marker. - PLoS One 9: e98607.

Daboussi, F., Leduc, S., Maréchal, A., Dubois, G., Guyot, V., PerezMichaut, C., Amato, A., Falciatore, A., Juillerat, A., Beurdeley, M., Voytas, D.F., Cavarec, L. \& Duchateau, P. (2014): Genome engineering empowers the diatom Phaeodactylum tricornutum for biotechnology. - Nat. Commun. 5: 3831.

Day, A. \& Goldschmidt-Clermont, M. (2011): The chloroplast transformation toolbox: selectable markers and marker removal. - Plant Biotechnol. J. 9: 540-553.

Doan, T.T.Y. \& Obbard, J.P. (2012): Enhanced intracellular lipid in Nannochloropsis $s p$. via random mutagenesis and flow cytometric cell sorting. - Algal Res. 1: 17-21.

Doan, Y.T.T. \& Obbard J.P. (2015): Two-stage cultivation of a Nannochloropsis mutant for biodiesel feedstock. - J. Appl. Phycol. 27: 2203-2208.

Doetsch, N.A., Favreau, M.R., Kuscuoglu, N., Thompson, M.D. \& Hallick, R.B. (2001): Chloroplast tranformation in Euglena gracilis: Splicing of a group III twintron transcribed from a transgenic $p s b K$ operon. - Curr. Genet. 39: 49-60.

Dong, H.P., Williams, E., Wang, D.Z., Xie, Z.X., Hsia, R.C., Jenck, A., Halden, R., Li, J., Chen, F. \& Place, A.R. (2013): Responses of Nannochloropsis oceanica IMET1 to long-term nitrogen starvation and recovery. - Plant Physiol. 162: 1110-1126.

Fang, X., Wei, C., Zhao-Ling, C. \& Fan, O. (2004): Effects of organic carbon sources on cell growth and eicosapentaenoic acid content of Nannochloropsis sp. - J. Appl. Phycol. 16: 499-503.

Fawley, M.W., Jameson, I. \& Fawley, K.P. (2015): The phylogeny of the genus Nannochloropsis (Monodopsidaceae, Eustigmatophyceae), with descriptions of $N$. australis $s p$. nov. and Microchloropsis gen. nov. - Phycologia 54: 545-552.

Fernandez-Reiriz, M.J. \& Labarta, U. (1996): Lipid classes and fatty acid composition of rotifers (Brachionus plicatilis) fed two algal diets. - Hydrobiologia 330: 73-79.

Ferreira, M., Coutinho, P., Seixas, P., Fabregas, J. \& Otero, A. (2009): Enriching Rotifers with "Premium" Microalgae. Nannochloropsis gaditana. - Mar. Biotechnol. 11: 585-595.

Franz, A.K., Danielewicz, M.A., Wong, D.M., Anderson, L.A. \& Boothe, J.R. (2013): Phenotypic screening with oleaginous microalgae reveals modulators of lipid productivity. - ACS Chem. Biol. 8: 1053-1062.

Gangl, D., Zedler, J.A.Z., Rajakumar, P.D., Ramos Martinez, E.M., Riseley, A., Włodarczyk, A., Purton, S., Sakuragi, Y., Howe, C.J., Jensen, P.E. \& Robinson, C. (2015): Biotechnological exploitation of microalgae. - J. Exp. Bot. 66: 6975-6990.

Georgianna, D.R., Hannon, M.J., Marcuschi, M., Wu, S., Botsch, K., Lewis, A.J., Hyun, J., Mendez, M. \& Mayfield, S.P. (2013): Production of recombinant enzymes in the marine alga Dunaliella tertiolecta. - Algal Res. 2: 2-9.

Gressel, J. (2013): Transgenic marine microalgae: a value-enhanced fishmeal and fish oil replacement. - In: Richmond, A. \& Hu, Q. (ed.), Handbook of Microalgal Culture: Applied Phycology and 
Biotechnology, pp. 653-670. - John Wiley \& Sons, Ltd., Chichester.

Gruber, A., Vugrinec, S., Hempel, F., Gould, S.B., Maier, U.G. \& Kroth, P.G. (2007): Protein targeting into complex diatom plastids: Functional characterisation of a specific targeting motif. Plant Mol. Biol. 64: 519-530.

Gutiérrez, C.L., Gimpel, J., Escobar, C., Marshall, S.H. \& Henríquez, V. (2012): Chloroplast genetic tool for the green microalgae Haematococcus pluvialis (Chlorophyceae, Volvocales). - J. Phycol. 48: 976-983.

Hannon, M., Gimpel, J., Tran, M., Rasala B. \& Mayfield, S. (2010): Biofuels from algae: challenges and potential. - Biofuels 1: 763-784

Hsu, P.D., Lander, E.S. \& Zhang, F. (2014): Development and applications of CRISPR-Cas9 for genome engineering. - Cell 157: $1262-1278$.

Hu, J., Wang, D., Li, J., Jing, G., Ning, K. \& Xu, J. (2014): Genomewide identification of transcription factors and transcriptionfactor binding sites in oleaginous microalgae Nannochloropsis. - Sci. Rep. 4: 5454.

Hu, Q., Xiang, W., Dai, S., Li, T., Yang, F., Jia, Q., Wang, G. \& $\mathrm{Wu}, \mathrm{H}$. (2015): The influence of cultivation period on growth and biodiesel properties of microalga Nannochloropsis gaditana 1049. - Bioresour. Technol. 192: 157-164.

Huang, Y.-J., Wang, L., Zheng, M.-G., Zheng, L., Tong, Y.-L. \& Li, Y. (2014): Overexpression of NgAUREO1, the gene coding for aurechrome 1 from Nannochloropsis gaditana, into Saccharomyces cerevisiae leads to a 1.6-fold increase in lipid accumulation. - Biotechnol. Lett. 36: 575-579.

Iwai, M., Hori, K., Sasaki-Sekimoto, Y., Shimojima, M. \& Ohta, H. (2015): Manipulation of oil synthesis in Nannochloropsis strain NIES-2145 with a phosphorus starvation-inducible promoter from Chlamydomonas reinhardtii. - Front. Microbiol. 6: 1-15.

Janouskovec, J., Horák, A., Oborník, M., Lukes, J. \& Keeling, P.J. (2010): A common red algal origin of the apicomplexan, dinoflagellate, and heterokont plastids. - Proc. Natl. Acad. Sci. U.S.A. 107: 10949-10954.

Jinkerson, R., Radakovits, R. \& Posewitz, M. (2013): Genomic insights from the oleaginous model alga Nannochloropsis gaditana. - Bioengineered 4: 37-43.

Kagan, M.L. \& Matulka, R.A. (2015): Safety assessment of the microalgae Nannochloropsis oculata. - Toxicol. Reports 2: $617-623$

Kagan, M.L., Sullivan, D.W., Gad, S.C. \& Ballou, C.M. (2014): Safety assessment of EPA-rich polar lipid oil produced from the microalgae Nannochloropsis oculata. - Int. J. Toxicol. 33: 459-474.

Kang, N.K., Choi, G., Kim, E.K., Shin, S., Jeon, S., Park, M.S., Jeong, K.J., Jeong, B., Chang, Y.K., Yang, J. \& Lee, B. (2015a): Heterologous overexpression of sfCherry fluorescent protein in Nannochloropsis salina. - Biotechnol. Reports 8: 10-15.

Kang, N.K., Jeon, S., Kwon, S., Koh, H.G., Shin, S.E., Lee, B., Choi, G.G., Yang, J.W., Jeong, B.R. \& Chang, Y.K. (2015b): Effects of overexpression of a bHLH transcription factor on biomass and lipid production in Nannochloropsis salina. Biotechnol. Biofuels 8: 200.

Kang, N.K., Lee, B., Shin, S.E., Jeon, S., Park, M.S. \& Yang, J.W. (2015c): Use of conditioned medium for efficient transformation and cost-effective cultivation of Nannochloropsis salina.Bioresource Technol. 181: 231-237.
Karas, B.J., Diner, R.E., Lefebvre, S.C., McQuaid, J., Phillips, A.P.R., Noddings, C.M., Brunson, J.K., Valas, R.E., Deerinck, T.J., Jablanovic, J., Gillard, J.T.F., Beeri, K., Ellisman, M.H., Glass, J.I., Hutchison, C.A. III, Smith, H.O., Venter, J.C., Allen, A.E., Dupont, C.L. \& Weyman, P.D. (2015): Designer diatom episomes delivered by bacterial conjugation. - Nat. Commun. 6 : 6925.

Kaye, Y., Grundman, O., Leu, S., Zarka, A., Zorin, B., Didi-Cohen, S., Khozin-Goldberg, I. \& Boussiba, S. (2015): Metabolic engineering toward enhanced LC-PUFA biosynthesis in Nannochloropsis oceanica: Overexpression of endogenous $\Delta 12$ desaturase driven by stress-inducible promoter leads to enhanced deposition of polyunsaturated fatty acids in TAG. - Algal Res. 11: 387-398.

Kilian, O., Benemann, C.S.E., Niyogi, K.K. \& Vick, B. (2011): High-efficiency homologous recombination in the oil-producing alga Nannochloropsis sp. - Proc. Natl. Acad. Sci. USA 108: 21265-21269.

Kindle, K.L., Richards, K.L. \& Stern, D.B. (1991): Engineering the chloroplast genome: techniques and capabilities for chloroplast transformation in Chlamydomonas reinhardtii. - Proc. Natl. Acad. Sci. USA 88: 1721-1725.

Klok, A.J., Lamers, P.P., Martens, D.E., Draaisma, R.B. \& Wijffels, R.H. (2014): Edible oils from microalgae: insights in TAG accumulation. - Trends Biotechnol. 32: 521-528.

Lapidot, M., Raveh, D., Sivan, A., Arad, S.M. \& Shapira, M. (2002): Stable chloroplast transformation of the unicellular red alga Porphyridium species. - Plant Physiol. 129: 7-12.

La Russa, M., Bogen, C., Uhmeyer, A., Doebbe, A., Filippone, E., Kruse, O. \& Mussgnug, J.H. (2012): Functional analysis of three type-2 DGAT homologue genes for triacylglycerol production in the green microalga Chlamydomonas reinhardtii. J. Biotechnol. 162: 13-20.

Lee, M.Y., Min, B.S., Chang. C.S. \& Jin E. (2006): Isolation and characterization of a xanthophyll aberrant mutant of the green alga Nannochloropsis oculata. - Mar. Biotechnol. (NY). 8: 238-245.

Lemahieu, C., Bruneel, C., Termote-Verhalle, R., Muylaert, K., Buyse, J. \& Foubert, I. (2013): Impact of feed supplementation with different omega-3 rich microalgae species on enrichment of eggs of laying hens. - Food Chem. 141: 4051-4059.

Li, F., Gao, D. \& Hu, H. (2014a): High-efficiency nuclear transformation of the oleaginous marine Nannochloropsis species using PCR product. - Biosci. Biotechnol. Biochem. 78: 812-817.

Li, J., Han, D., Wang, D., Ning, K., Jia, J., Wei, L., Jing, X., Huang, S., Chen, J., Li, Y., Hu, Q. \& Xu, J. (2014b): Choreography of transcriptomes and lipidomes of Nannochloropsis reveals the mechanisms of oil synthesis in microalgae. - Plant Cell 26: $1645-1665$.

Li, S.S. \& Tsai, H.J. (2009): Transgenic microalgae as a nonantibiotic bactericide producer to defend against bacterial pathogen infection in the fish digestive tract. - Fish Shellfish Immunol. 26: 316-325.

Lu, Y. \& Xu, J. (2015): Phytohormones in microalgae: a new opportunity for microalgal biotechnology? - Trends Plant Sci. 20: 273-282.

Lubián, L.M. (1982): Nannochloropsis gaditana sp. nov., a new marine Eustigmatophyceae. - Lazaroa 4: 287-293.

Lubzens, E., Gibson, O., Zmora, O. \& Sukenik, A. (1995): Potential advantages of frozen algae (Nannochloropsis $s p$.) for rotifer (Brachionus plicatilis) culture. - Aquaculture 133: 295-309. 
Ma, X.N., Chen, T.P., Yang, B., Liu J. \& Chen, F. (2016): Lipid production from Nannochloropsis. - Marine Drugs 14: 61.

Ma, Y., Wang, Z., Yu, C., Yin, Y. \& Zhou, G. (2014): Evaluation of the potential of 9 Nannochloropsis strains for biodiesel production. - Bioresour. Technol. 167: 503-509.

Ma, Y., Wang, Z., Zhu, M., Yu, C., Cao, Y., Zhang, D. \& Zhou, G. (2013): Increased lipid productivity and TAG content in Nannochloropsis by heavy-ion irradiation mutagenesis. Bioresour. Technol. 136: 360-367.

Medipally, S.R., Yusoff, F.M., Banerjee, S. \& Shariff, M. (2015): Microalgae as sustainable renewable energy feedstock for biofuel production. - Biomed. Res. Int. 2015: 519513.

Moog, D., Stork, S., Reislöhner, S., Grosche, C. \& Maier, U.-G. (2015): In vivo localization studies in the Stramenopile alga Nannochloropsis oceanica. - Protist 166: 161-171.

Mühlroth, A., Li, K., Røkke, G., Winge, P., Olsen, Y., HohmannMarriott, M.F., Vadstein, O. \& Bones, A.M. (2013): Pathways of lipid metabolism in marine algae, co-expression network, bottlenecks and candidate genes for enhanced production of EPA and DHA in species of Chromista. - Mar. Drugs 11: 4662-4697.

Murakami, R. \& Hashimoto, H. (2009): Unusual nuclear division in Nannochloropsis oculata (Eustigmatophyceae, Heterokonta) which may ensure faithful transmission of secondary plastids. Protist 160: 41-49.

Nymark, M., Sharma, A.K., Sparstad, T., Bones, A.M. \& Winge, P. (2016): A CRISPR/Cas9 system adapted for gene editing in marine algae. - Sci. Rep. 6: 24951.

O'Brien, J.A. \& Lummis, S.C.R. (2011): Nano-biolistics: a method of biolistic transfection of cells and tissues using a gene gun with novel nanometer-sized projectiles. - BMC Biotechnol. 11: 66.

Pan, K., Qin, J., Li, S., Dai, W., Zhu, B., Jin, Y., Yu, W., Yang, G. \& Li, D. (2011): Nuclear monoploidy and asexual propagation of Nannochloropsis oceanica (Eustigmatophyceae) as revealed by its genome sequence. - J. Phycol. 47: 1425-1432.

Perin, G., Bellan, A., Segalla, A., Meneghesso, A., Alboresi, A. \& Morosinotto, T. (2015): Generation of random mutants to improve light-use efficiency of Nannochloropsis gaditana cultures for biofuel production. - Biotechnol. Biofuels 8: 161.

Potvin, G. \& Zhang, Z. (2010): Strategies for high-level recombinant protein expression in transgenic microalgae: A review. Biotechnol. Adv. 28: 910-918.

Purton, S., Szaub, J.B., Wannathong, T., Young, R, \& Economou, C.K. (2013): Genetic engineering of algal chloroplasts: progress and prospects. - Rus. J. Plant Physiol. 60: 491-499.

Radakovits, R., Jinkerson, R.E., Fuerstenberg, S.I., Tae, H., Settlage, R.E., Boore, J.L. \& Posewitz, M.C. (2012): Draft genome sequence and genetic transformation of the oleaginous alga Nannochloropis gaditana. - Nat. Commun. 3: 686.

Schneider, J.C., Livne, A., Sukenik, A. \& Roessler, P.G. (1995): A mutant of Nannochloropsis deficient in eicosapentaenoic acid production. - Phytochemistry 40: 807-814.

Sharma, K. \& Schenk, P.M. (2015): Rapid induction of omega-3 fatty acids (EPA) in Nannochloropsis sp. by UV-C radiation. Biotechnol. Bioeng. 112: 1243-1249.

Shih, C.-H., Chen, H.-Y., Lee, H.-C. \& Tsai, H.-J. (2015): Purple chromoprotein gene serves as a new selection marker for transgenesis of the microalga Nannochloropsis oculata. - PLoS One 10: e0120780.
Simionato, D., Block, M.A., La Rocca, N., Jouhet, J., Maréchal, E., Finazzi, G. \& Morosinotto, T. (2013): The response of Nannochloropsis gaditana to nitrogen starvation includes de novo biosynthesis of triacylglycerols, a decrease of chloroplast galactolipids, and reorganization of the photosynthetic apparatus. - Eukaryot. Cell 12: 665-676.

Şirin, S. \& Sillanpää, M. (2015): Cultivating and harvesting of marine alga Nannochloropsis oculata in local municipal wastewater for biodiesel. - Bioresour. Technol. 191: 79-87.

Siripornadulsil, S., Dabrowski, K. \& Sayre, R. (2007): Microalgal vaccines. - Adv. Exp. Med. Biol. 616: 122-128.

Sorigué, D., Légeret, B., Cuiné, S., Morales, P., Mirabella, B., Guédeney, G., Li-Beisson, Y., Jetter, R., Peltier, G. \& Beisson, F. (2016): Microalgae synthesize hydrocarbons from long-chain fatty acids via a light-dependent pathway. - Plant Physiol. 171: 2393-2405.

Starkenburg, S.R., Kwon, K.J., Jha, R.K., McKay, C., Jacobs, M., Chertkov, O., Twary, S., Rocap, G. \& Cattolico, R. (2014): A pangenomic analysis of the Nannochloropsis organellar genomes reveals novel genetic variations in key metabolic genes. - BMC Genomics 15: 212.

Su, C.H., Chien, L.J., Gomes, J., Lin, Y.S., Yu, Y.K., Liou, J.S. \& Syu, R.J. (2011): Factors affecting lipid accumulation by Nannochloropsis oculata in a two-stage cultivation process. J. Appl. Phycol. 23: 903-908.

Sukenik, A. (1999): Production of eicosapentaenoic acid by the marine eustigmatophyte Nannochloropsis. - In: Cohen, Z. (ed.), Chemicals from Microalgae, pp. 41-53. - Taylor and Francis, London.

Sunaga, Y., Maeda, Y., Yabuuchi, T., Muto, M., Yoshino, T. \& Tanaka, T. (2014): Chloroplast-targeting protein expression in the oleaginous diatom Fistulifera solaris JPCC DA0580 toward metabolic engineering. - J. Biosci. Bioeng. 119: 28-34.

Taleb, A., Pruvost, J., Legrand, J., Marec, H., Le-Gouic, B., Mirabella, B., Legeret, B., Bouvet, S., Peltier, G., Li-Beisson, Y., Taha, S. \& Takache, H. (2015): Development and validation of a screening procedure of microalgae for biodiesel production: application to the genus of marine microalgae Nannochloropsis. Bioresource Technol. 177: 224-232.

Tian, J., Zheng, M., Yang, G., Zheng, L., Chen, J. \& Yang, B. (2013): Cloning and stress-responding expression analysis of malonyl CoA-acyl carrier protein transacylase gene of Nannochloropsis gaditana. - Gene 530: 33-38.

Umdu, E.S., Tuncer, M. \& Seker, E. (2009): Transesterification of Nannochloropsis oculata microalga's lipid to biodiesel on $\mathrm{Al}_{2} \mathrm{O}_{3}$ supported $\mathrm{CaO}$ and $\mathrm{MgO}$ catalysts. - Bioresour. Technol. 100: 2828-2831.

van den Hoek, C., Mann, D.G. \& Jahns, H.M. (1995): Algae: An Introduction to Phycology. Cambridge University Press, Cambridge, UK.

Vieler, A., Wu, G., Tsai, C.-H., Bullard, B., Cornish, A.J., Harvey, C., Reca, I.-B., Thornburg, C., Achawanantakun, R., Buehl, C.J., Campbell, M.S., Cavalier, D., Childs, K.L., Clark, T.J., Deshpande, R., Erickson, E., Armenia Ferguson, A., Handee, W., Kong, Q., Li, X., Liu, B., Lundback, S., Peng, C., Roston, R.L., Sanjaya, Simpson, J.P., TerBush, A., Warakanont, J., Zäuner, S., Farre, E.M., Hegg, E.L., Jiang, N., Kuo, M.-H., Lu, Y., Niyogi, K.K., Ohlrogge, J., Osteryoung, K.W., Shachar-Hill, Y., Sears, B.B., Sun, Y., Takahashi, H., Yandell, M., Shiu, S.-H. \& Benning, C. (2012): Genome, functional gene annotation, and nuclear transformation of the heterokont oleaginous alga 
Nannochloropsis oceanica CCMP1779. - PLoS Genet 8: e1003064.

Vree, J.H. de, Bosma, R., Janssen, M., Barbosa, M.J. \& Wijffels, R.H. (2015): Comparison of four outdoor pilot-scale photobioreactors. - Biotechnol. Biofuels 8: 215.

Wang, D., Ning, K., Li, J., Hu, J., Han, D., Wang, H., Zeng, X., Jing, X., Zhou, Q., Su, X., Chang, X., Wang, A., Wang, W., Jia, J., Wei, L., Xin, Y., Qiao, Y., Huang, R., Chen, J., Han, B., Yoon, K., Hill, R.T., Zohar, Y., Chen, F., Hu, Q. \& Xu, J. (2014): Nannochloropsis genomes reveal evolution of microalgal oleaginous traits. - PLoS Genet. 10: e1004094.

Weeks, D.P. (2011): Homologous recombination in Nannochloropsis: a powerful tool in an industrially relevant alga. - Proc. Natl Acad. Sci. USA 108: 20859-20860.

Wei, L., Xin, Y., Wang, D., Jing, X., Zhou, Q., Su, X., Jia, J., Ning, K., Chen, F., Hu, Q. \& Xu, J. (2013): Nannochloropsis plastid and mitochondrial phylogenomes reveal organelle diversification mechanism and intragenus phylotyping strategy in microalgae. - BMC Genomics 14: 534.

Weyman, P.D., Beeri, K., Lefebvre, S.C., Rivera, J., McCarthy, J.K., Heuberger, A.L., Peers, G., Allen, A.E. \& Dupont, C.L. (2015): Inactivation of Phaeodactylum tricornutum urease gene using transcription activator-like effector nuclease-based targeted mutagenesis. - Plant Biotechnol. J. 13: 460-470.

Xie, W.H., Zhu, C.C., Zhang, N.S., Li, D., Yang, W.D., Liu, J.S., Sathishkumar, R. \& Li, H.Y. (2014): Construction of novel chloroplast expression vector and development of an efficient transformation system for the diatom Phaeodactylum tricornutum. Mar. Biotechnol. (NY) 16: 538-546.
Zhang, C. \& Hu, H. (2013): High-efficiency nuclear transformation of the diatom Phaeodactylum tricornutum by electroporation. Mar. Genomics 16: 63-66.

Zheng, M.G., Huang, Y.J., Zheng, L., Sun, Z.T. \& Wang, L. (2014): Cloning, expression and stress-respondent transcription of longchain acyl-coenzyme A synthetase cDNA gene of Nannochloropsis gaditana and its involvement in the biosynthesis of eicosapentaenoic and decosahexaenoic acids. - Biotechnol. Lett. 36: 141-145.

Zheng, M., Tian, J., Yang, G., Zheng, L., Chen, G., Chen, J. \& Wang, B. (2013): Transcriptome sequencing, annotation and expression analysis of Nannochloropsis $s p$. at different growth phases. - Gene 523: 117-121.

Zhu, B., Sun, F., Yang, M., Lu, L., Yang, G. \& Pan, K. (2014): Large-scale biodiesel production using flue gas from coal-fired power plants with Nannochloropsis microalgal biomass in open raceway ponds. - Bioresour. Technol. 174: 53-59.

Manuscript received: 9 May 2016

Accepted: 9 September 2016

Handling editor: Burkhard Becker 